\title{
Study of left and right ventricular function in Romano-Ward syndrome
}

Stephen J Cross, John C S Dean, Hai Shiang Lee, Murdoch Y Norton, Noel T S Evans, Francis Smith, Kevin Jennings, Stephen Walton inherited condition with autosomal dominant transmission. ${ }^{3}$

It is proposed that there is an imbalance of the sympathetic innervation to the heart with a relative left sided over activity. ${ }^{4}$

Postmortem reports in some of these patients have found evidence of neuritis and myocarditis. ${ }^{56}$ This has prompted us to examine, in vivo, left and right ventricular function in members of two families with this condition.

Setting-Department of Nuclear Medicine, Aberdeen Royal Infirmary.

Patients-Eight subjects from two families known to have Romano-Ward syndrome, four of whom (two from each family) had had symptoms.

Results-The five subjects from family 1 had normal left ventricular contraction; two had subtle abnormalities of right ventricular phase. In family 2 all three subjects had abnormal left ventricular contraction (reduced amplitude in three, abnormal phase in two). All had subtle abnormalities of right ventricular phase.

Conclusion-Abnormal right or left ventricular myocardium may be partly or wholly responsible for the repolarisation changes seen on the electrocardiogram of these families or may act as an ectopic focus to start ventricular tachycardias in a susceptible heart.

(Br Heart f 1993;70:266-271)

Romano-Ward syndrome is characterised by a prolonged QT interval and the propensity to recurrent ventricular arrhythmias that may precipitate syncope or sudden death. ${ }^{12}$ It is an

\section{Accepted for publication 18 January 1993 \\ Department of \\ Department of Medical Genetics \\ Department of Medical Physics N T S Evans \\ Department of Nuclear Medicine, Infirmary \\ F Smith \\ Correspondence to: Dr S J Cross, Department of Cardiology, Aberdeen Royal Infirmary, Foresterhill,}

The study was approved by the local ethics

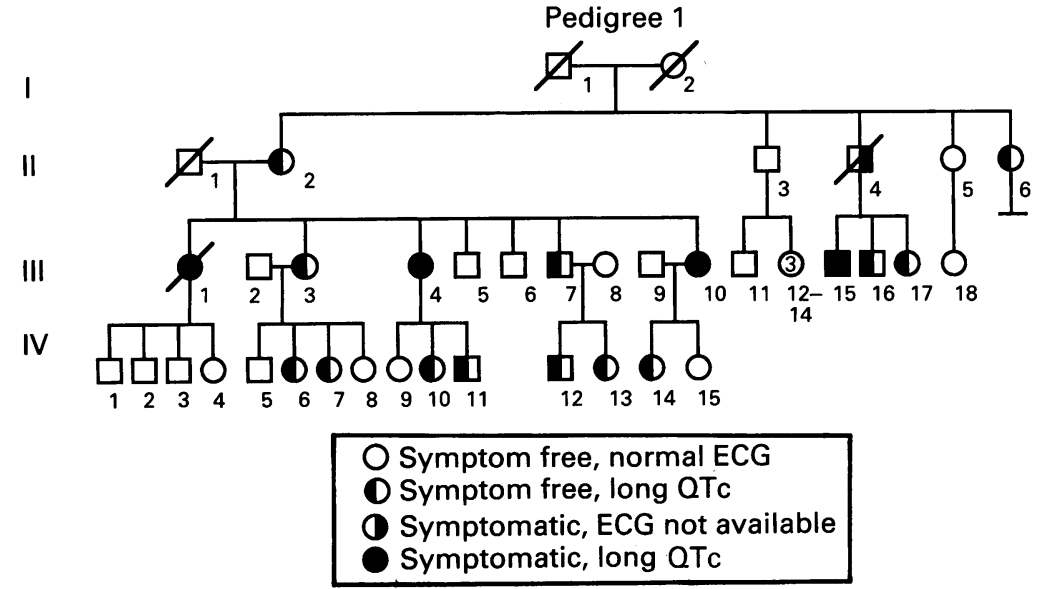

Figure 1 Pedigree of family 1. No electrocardiograms were available for I.1, I.2 and II.4 as they died before the start of the study.

\section{Patients and methods} committee. Informed consent was obtained from all patients.

\section{ELECTROCARDIOGRAMS}

The electrocardiograms taken for screening of symptom free relatives were recorded when the subjects were not receiving any treatment known to affect the QT interval. The QTc intervals were calculated with Bazett's formula. ${ }^{7}$ The QTc intervals quoted in the tables are the longest that have been recorded on any occasion for that patient, and the values of QT and resting heart rates quoted correspond to these electrocardiograms. A value of QTc above $440 \mathrm{~ms}$ is considered to be prolonged. The $U$ waves were differentiated from $T$ waves by the method described by Lepeschkin. ${ }^{8}$

\section{STUDY PATIENTS}

Family 1

This family first came to medical attention when subject III.4 presented with a pseudoepileptiform seizure. A biochemical profile, electroencephalogram, computed tomography scan of the brain, and echocardiography were all normal. A resting electrocardiogram, however, showed a prolonged QTc interval of $0.47 \mathrm{~s}$. Holter monitoring showed episodes of ventricular bigeminy. Cardiac catheterisation (performed because of exertional chest pain) showed normal coronary arteries. The left ventriculogram was normal. Treatment with propranolol abolished the pseudoseizures.

Subject III.10 presented independently at the age of 32 with an epileptiform seizure during a severe exacerbation of duodenal ulcer disease complicated by duodenal stenosis. Biochemical profile at presentation showed hypokalaemia $(2 \cdot 4 \mathrm{mmol} / \mathrm{l})$. Her QTc was prolonged $(0.54 \mathrm{~s})$ and remained so after correction of the electrolyte disturbance.

Family screening showed 15 other subjects with abnormal electrocardiograms (fig 1). 
Table 1 Demographic data of members of family 1 aged over 18

\begin{tabular}{llllllll}
\hline Patient & Age $(y r)$ & Sex & Heart rate & $Q T(s)$ & $Q T c(s)$ & Symptoms & $R N V$ \\
\hline II.2 & 69 & F & 64 & 0.60 & 0.62 & - & - \\
II.6 & 56 & F & 48 & 0.54 & 0.57 & - & - \\
III.1 & 47 & F & 64 & 0.60 & 0.62 & + & - \\
III.3 & 45 & F & 53 & 0.64 & 0.60 & - & + \\
III.4 & 44 & F & 73 & 0.58 & 0.64 & + & + \\
III.7 & 36 & M & 80 & 0.54 & 0.62 & - & + \\
III.10 & 33 & F & 75 & 0.56 & 0.63 & + & + \\
III.15 & 33 & M & 70 & 0.58 & 0.63 & + & - \\
III.16 & 30 & M & 48 & 0.62 & 0.55 & - & - \\
III.17 & 27 & F & 103 & 0.38 & 0.50 & - & + \\
IV.6 & 27 & F & 60 & 0.64 & 0.64 & - & - \\
IV.7 & 25 & F & 64 & 0.62 & 0.64 & - & - \\
IV.10 & 22 & F & 83 & 0.40 & 0.47 & - & - \\
\hline
\end{tabular}

$\mathrm{RNV}$, radionuclide ventriculography; QTc is maximum recorded at any time for each subject.

Table 1 shows the demographic details of the 13 affected adults assessed.

Subject III. 1 died suddenly at home shortly after diagnosis. She was aged 46 , and had been receiving diuretic treatment for mild hypertension, but her biochemical profile was normal. One night she felt unwell, rose from her bed, and collapsed and died. A detailed postmortem examination found no abnormalities. It was concluded that an arrhythmia had been responsible for the death.

Subject III.15 had one episode of syncope after two episodes of near syncope at the age of 18 , while under severe physical stress.

Other family members have been symptom

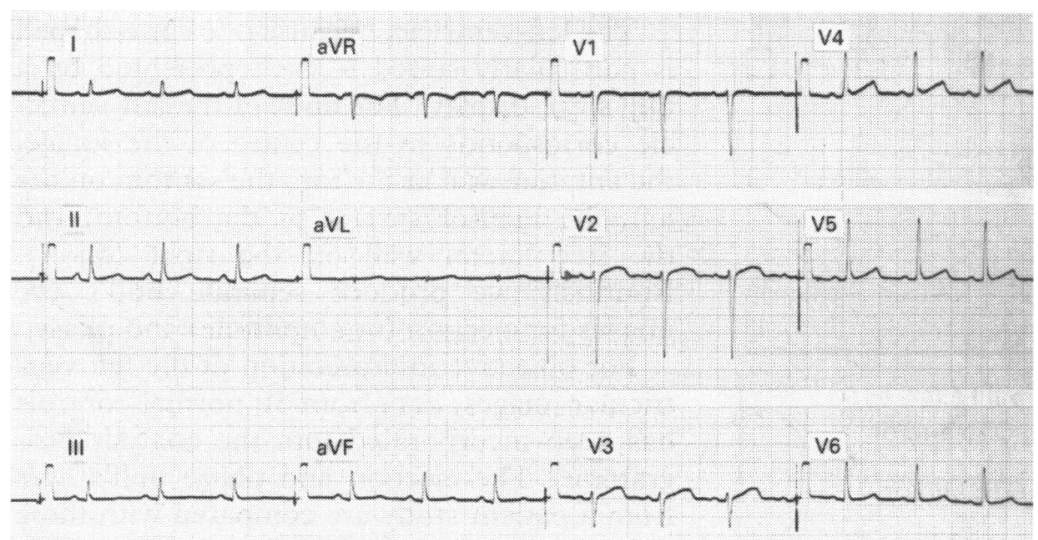

Figure 2 Electrocardiogram from III.7 (family 1) showing prolonged QTc at 0.50 s, and abnormal ST segment configuration. Patient was not receiving treatment.

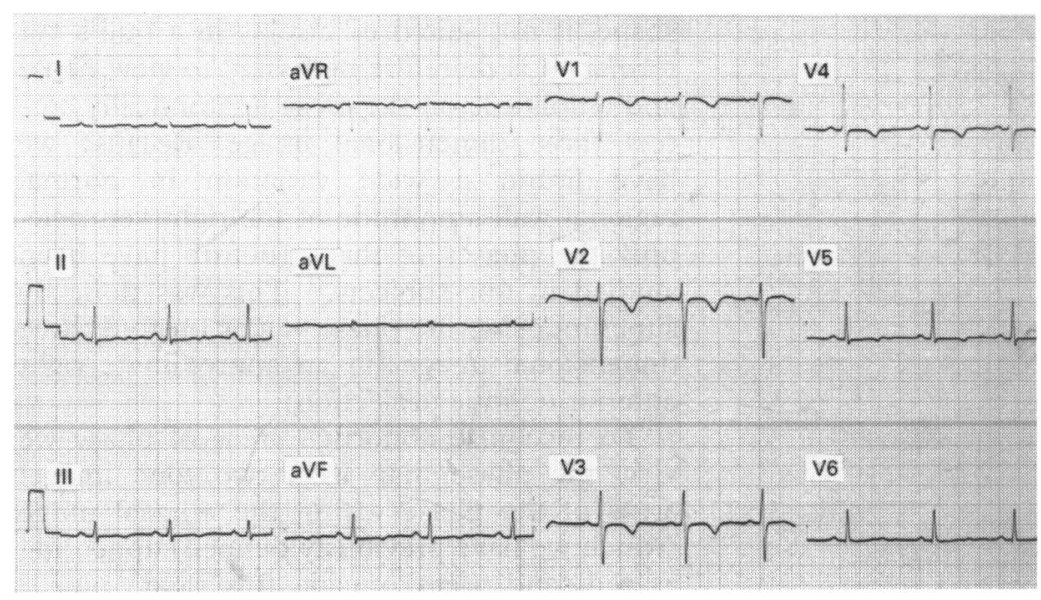

Figure 3 Electrocardiogram from III.17 (family 1) showing prolonged QTc at $0.47 \mathrm{~s}$, and inverted or biphasic $T$ waves in the chest leads. Patient was not receiving treatment. free, with the exception of II.4 who is reported to have had one seizure after being startled at the age of 6 . Unfortunately, no electrocardiogram is available as he died some years ago from malignancy.

The electrocardiographic findings in this family take one of three forms: ST changes with QT prolongation (fig 2), inverted or bifid $T$ waves, or both, with prolongation of the QT interval (fig 3), or normal ST segment $T$ wave morphology with prolongation of the QT interval. There have been no recorded episodes of $T$ wave alternans. Invasive electrophysiology has not been undertaken in any member of this family.

Of the 12 living members who are over 18 years of age, five agreed to take part in the research studies: two of these have been symptomatic.

\section{Family 2}

This family first came to medical attention when IV.2 presented with focal convulsions at the age of 31 years. A computed tomography scan of the brain was performed, which was reported to be normal. Treatment with phenytoin for presumed epilepsy was begun.

Five years later, at a routine school medical examination, V.1 (daughter of IV.2) was found to have an irregular pulse. On direct questioning she complained of some shortness of breath on cross country running, episodes of dizziness, and presyncope. There had not been any episodes of loss of consciousness. An electrocardiogram showed underlying sinus rhythm and ventricular extra systoles with runs of non-sustained polymorphic ventricular tachycardia, which were also seen on a 24 hour Holter recording (fig 4). The QTc interval at this stage was normal, but there were prominent $U$ waves, especially in the right chest leads.

An invasive electrophysiology study, performed at another centre, showed normal sinus node function, functional duality of the atrioventricular node conduction, a normal $\mathrm{HV}$ interval, and normal ventricular refractory periods. Aggressive stimulation in multiple right ventricular sites failed to provoke a sustained arrhythmia. A Valsalva manoeuvre produced a flat heart rate response, did not cause any elecrocardiographic changes, and did not precipitate an arrhythmia. Holter monitoring showed a break in the arrhythmias overnight, with an early morning resurgence.

An electrocardiogram taken from IV.2 at this time showed considerable repolarisation changes (similar to those of her daughter) and a period of non-sustained ventricular tachycardia. The clinical and electrophysiological findings were considered consistent with Romano-Ward syndrome.

Permanent pacing, with additional $\beta$ blockade in the case of V.1, has been a successful treatment in these two patients, stopping all palpitations and blackouts.

Electrocardiograms were obtained from the other members of the family, none of whom have any impairment of their hearing. A total 


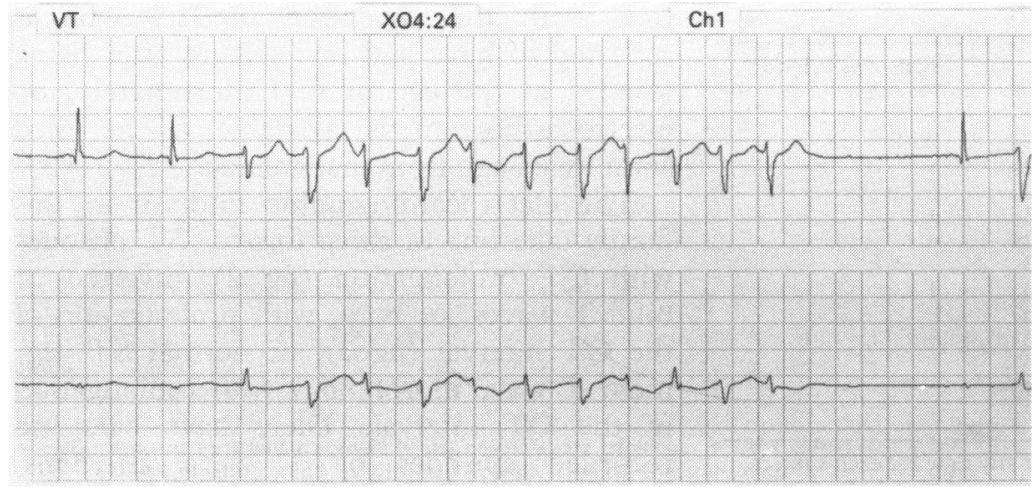

Figure 4 Recording from a 2 channel 24 hour electrocardiogram from individual $V .1$ (family 2) showing a self limiting run of multiform ventricular tachycardia.

of five members of this family are considered to have Romano-Ward syndrome (fig 5). These five have prominent $U$ waves on their electrocardiograms (fig 6), some have prolonged QT and QTc intervals, and some have a sinus bradycardia (table 2). No episodes of

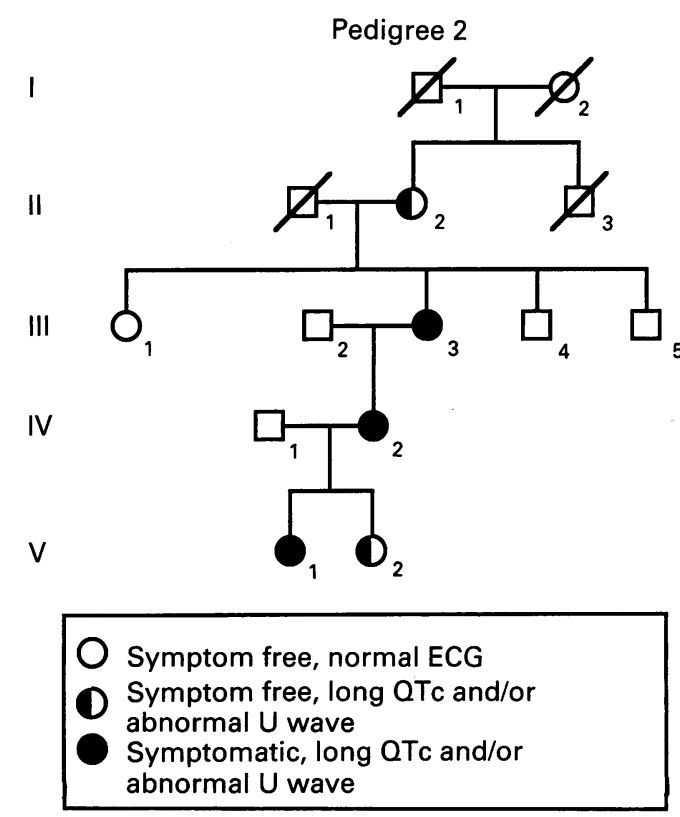

Figure 5 Pedigree of family 2. No electrocardiograms were available for I.1, I.2, II.1, and II. 3 as they died before the start of the study.

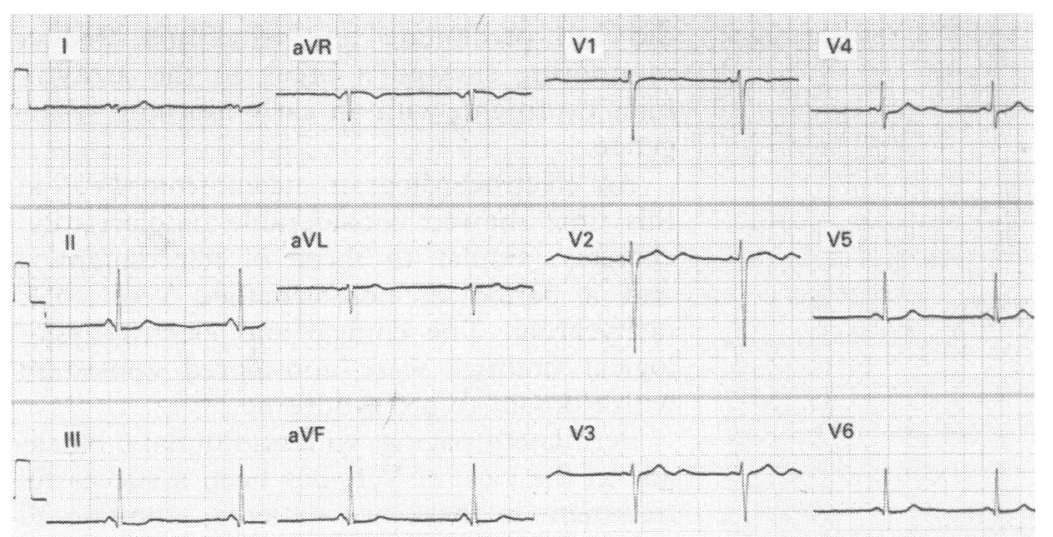

Figure 6 Electrocardiogram from affected member (V.2) of family 2 showing sinus bradycardia, a QTc of $0.42 \mathrm{~s}$, and abnormally prominent $U$ waves. Patient was not receiving medication.
$T$ wave alternans has been recorded. In this family there have not been any sudden unexplained deaths.

TOMOGRAPHIC RADIONUCLIDE VENTRICULOGRAPHY

The blood pool was labelled conventionally with sequential injections of $7.5 \mathrm{mg}$ stannous pyrophosphate and $800 \mathrm{MBq}$ of technetium$99 \mathrm{~m}$. Tomography of the cardiac blood pool was then undertaken with the Aberdeen section scanner.

This scanner has been described elsewhere, ${ }^{10}$ but in summary it is a gated, focused tomographic scanner that uses six transaxial slices, measured sequentially, to image the heart in less than 20 minutes. These individual slices are interpolated together to produce a three dimensional data image of the ventricular blood pool, at each of eight phases through the cardiac cycle. Amplitude, ejection fraction, and phase maps can be superimposed on this image, which can be orientated to view any aspect of the cardiac blood pool.

At the time of image reconstruction the mean $R R$ interval during acquisition of the data is calculated; only data from those cycles with RR intervals between $60 \%$ and $140 \%$ of this value are included in the final construction of the image. Hence, extrasystoles are not included in the final analysis.

The left ventricle, because it is cone shaped is particularly suited to be represented by a bull's eye display. The apex of the left ventricle corresponds to the centre of the image, the anterior wall at the top, the septum on the left, the inferior surface at the bottom, and the free lateral wall on the right (fig 7). Routinely we produce separate bull's eye images for ejection (or amplitude) and phase.

For objective interpretation of the left ventricular images, data from 50 normal controls has been incorporated into the analysis programme. The ejection and phase bull's eyes from a patient study are compared with these controls, and abnormal areas (outside 2 SDs) are displayed on separate corresponding error maps (fig 8). Normal regions of left ventricle are depicted as white areas on these error maps.

The right ventricle, because of its crescent shape, is not suited to display in a bull's eye format. It is therefore necessary to view phase and amplitude on the more anatomically correct three dimensional image. Because we have found a wide variation in normal regional wall amplitude of the right ventricle, only the phases of this structure have been studied in our subjects. ${ }^{11}$ This was done by superimposing the phase values on to a three dimensional image (in an approximate right anterior oblique projection).

To recognise abnormal areas of phase we view the phase map as a two tone image: areas of the phase within the normal range (which we have previously defined) appearing as red, and areas outside this range blue. ${ }^{12}$ This, once again, allowed objective assessment of the images. 
Table 2 Demographic data of affected members of family 2

\begin{tabular}{lllllllll}
\hline Patient & Age $(y r)$ & Sex & Heart rate & $Q T(s)$ & $Q T c(s)$ & Symptoms & $R N V$ & $E F(\%)$ \\
\hline II.2 & 86 & F & 61 & 0.45 & 0.45 & - & - & - \\
III.3 & 64 & F & 58 & 0.48 & 0.49 & + & - & - \\
IV.2 & 42 & F & 38 & 0.34 & 0.43 & + & + & 27 \\
V.1 & 19 & F & 45 & 0.41 & 0.47 & + & + & 26 \\
V.2 & 18 & F & 71 & 0.46 & 0.42 & - & + & 38 \\
\hline
\end{tabular}

$\mathrm{RNV}$, radionuclide ventriculography; EF, ejection fraction; QTc is maximum recorded at any time for each subject.

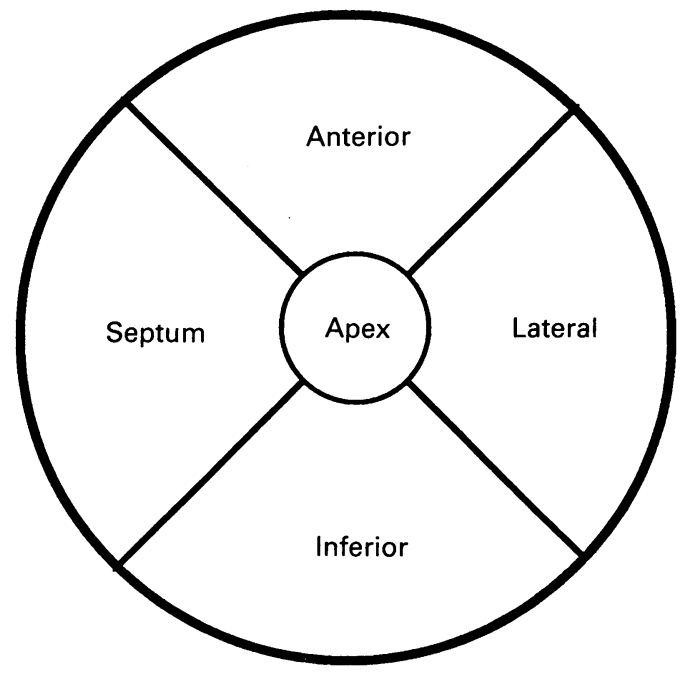

Figure 7 Regions of the left ventricle depicted as a bull's eye image.

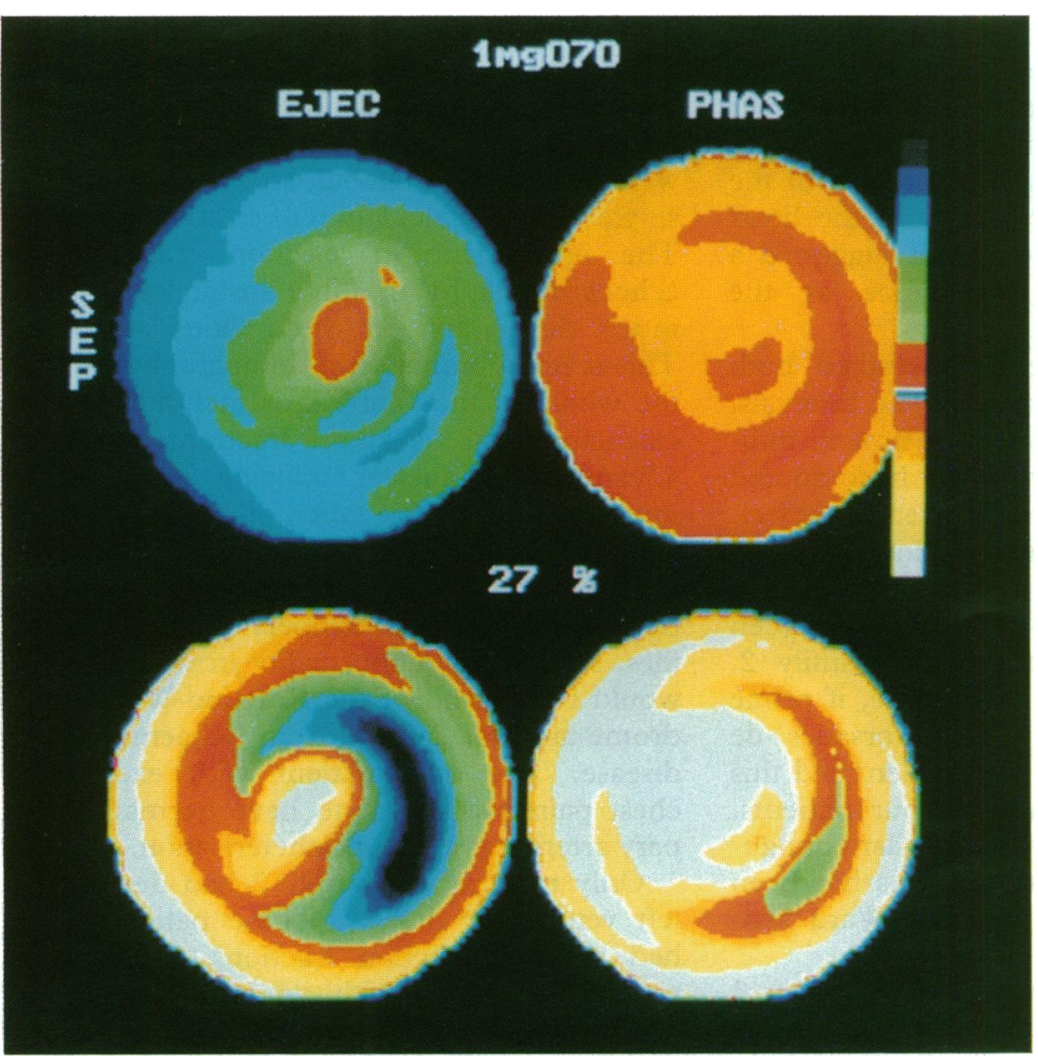

Figure 8 Bull's eye image of left ventricular ejection and phase with corresponding error maps for patient IV.2 (family 2). There is reduced ejection in the anterior, inferior, and lateral regions. The ejection fraction is reduced (normal $>40 \%$ ). The phase is abnormal in the inferior and lateral regions. Normal areas on the error maps are coloured white.
Alternatively, the phase of either ventricle can be studied by superimposing a dynamic phase wave on to the three dimensional image, and viewing the changes in phase in real time. Although this technique is subjective, we have found excellent between observer agreement (unpublished data). When the data images are reanalysed, the values of ejection and phase are consistent (unpublished data).

During acquisition of the scans, patients IV.2 and V.1 had the rates of their pacemakers reduced so that their underlying (sinus) rhythm was present.

\section{Results}

LEFT VENTRICULAR FUNCTION

All three members of family 2 had abnormal left ventricular function. The mother (IV.2) had a large area, covering most of the left ventricle, of reduced ejection with a matching phase abnormality (fig 8). The ejection fraction was reduced at $27 \%$ (normal $>40 \%$ ). Ejection is linearly related to amplitude, but is not dependent on the number of counts for an absolute value.

The symptomatic daughter (V.1) had a reduced area of ejection in the inferolateral and apical regions of the left ventricle, but without any phase abnormality. The ejection fraction was reduced at $26 \%$. The symptom free daughter (V.2) had a small matching area of reduced amplitude and abnormal phase in the inferolateral region of the left ventricle. The ejection fraction was minimally reduced (38\%).

The scans of family 1 did not show any areas of abnormal left ventricular contraction: they all had normal ejection fractions.

\section{RIGHT VENTRICULAR FUNCTION}

The findings of the right ventricular phases were not uniform. Whereas all subjects had values of right ventricular phases within the normal range, the dynamic phase wave was sometimes abnormal.

In family 2, V.1 and V.2 had abnormal phase waves in that they started in the middle part of the anterior free wall of the right ventricle instead of towards the right ventricular apex. In IV.2 the phase wave started in the normal region but its progress was delayed and persistent at a small area towards the right ventricular outflow tract.

In family 1, III.17 and III.7 the phase wave started in the normal area but progress was delayed and persistent in a small area at the 
posterior aspect of the right ventricular apex. The location of these abnormal areas was identical for these two subjects.

The other members of family 1 had normal right ventricular scans.

The results of the radionuclide angiography prompted us to perform echocardiography on the two symptomatic patients from family 2 . Both of these subjects had reduced motion in the apical region of their left ventricles. The ejection fraction, from a single view in the parasternal short axis orientation, was, however, in the normal range. Right ventricular function seemed normal.

\section{Discussion}

Romano-Ward syndrome is a rare hereditary disorder characterised by a prolonged QT interval and a propensity to ventricular tachycardias that may precipitate sudden death. ${ }^{12}$ The prolonged QT interval reflects delayed ventricular repolarisation. This state may predispose to arrhythmias after ventricular extrasystoles.

Autonomic neuropathy in diabetic patients can produce QT prolongation, ${ }^{13}$ as can right stellate ganglionectomy or left stellate stimulation in animal models. ${ }^{14}$ It has therefore been inferred that autonomic dysfunction is the most likely mechanism of QT prolongation in Romano-Ward syndrome.

Postmortem studies performed in some patients with Romano-Ward syndrome have shown focal areas of neuritis and myocarditis. ${ }^{56}$

It has been proposed that an intracardiac abnormality, instead of a neural defect, could decrease electrical stability and make the myocardium more vulnerable to sympathetic discharges. ${ }^{15}$ A cellular mechanism has been suggested to explain such a susceptibility. ${ }^{16}$

There has been a single case report of the findings of electron microscopy in the heart of a Romano-Ward patient; this suggested a myocardial metabolic disturbance as the underlying defect. ${ }^{17}$

The two families we have studied both have members with prolonged QTc intervals. The QTc interval can vary with time in individual subjects. ${ }^{718}$ Therefore we have quoted the highest values obtained for each subject. Propranolol can affect the QTc interval, typically shortening it. ${ }^{19} 20$

Multiform ventricular tachycardia has been documented in two members of family 2 . Patients with a long QT syndrome, if prone to arrhythmias, typically have torsades de pointes. Although we have not identified this specific tachycardia in any of our patients, ventricular tachycardia with a similar configuration to that seen in our subjects has been described previously in Romano-Ward syndrome. ${ }^{21} 22$

The prominent $U$ wave seen in family 2 does not seem to be dependent on the heart rate: sinus bradycardia was not present in V.1.

In family 1 an apparently healthy member (III.1) died suddenly, presumably from an arrhythmia. The presence of hypokalaemia in individual III. 10 in association with her pseudoseizure may be important, as it is known that hypokalaemia prolongs the QT interval, ${ }^{23}$ and this is thought to increase the tendency to ventricular arrhythmia in those with a congenital prolongation of the QT interval. Precipitation of arrhythmias by physical exertion has been described previously in Romano-Ward syndrome, ${ }^{4}$ and this may be relevant to the experience of III.15.

Both families have normal hearing, and the family trees suggest an autosomal dominant inheritance: the affected members of these families therefore have Romano-Ward syndrome.

Romano-Ward syndrome does not have the same importance for each affected person: frequency of symptoms can vary from many syncope attacks a month to a few each year, or only one or two episodes during the whole of the subject's life. ${ }^{4}$

The Aberdeen section scanner has been shown to be a sensitive method of detecting regional abnormalities in wall motion. It is more sensitive in this respect than conventional planar techniques, especially for imaging the inferior surface of the left ventricle. ${ }^{24} 25$ Although the images of our subjects were not interpreted blinded, analysis of left ventricular phase and amplitude is objective because of the use of error images. Similarly the three dimensional display of values of right ventricular phase is objective, whereas the dynamic display of spread of left or right ventricular phase is subjective.

The left ventricular wall motion as assessed by echocardiography in IV.2 and V.1 in family 2 confirm the findings from radionuclide angiography. The normal ejection fractions measured with ultrasound were not surprising as the wall motion abnormalities on echo were local and not global, and we only used a single view for calculation of ejection fraction. The assessment of ejection fraction by echocardiography in these instances is very much dependent on the regions sampled. This is not a limitation of tomography, where the whole of the ventricle is included in the calculation of ejection fraction. Another advantage of nuclear imaging is that the images obtained are not dependent on the subject having a good acoustic window.

It was important to restrict our investigations to the younger members of the two families, so that any abnormality in wall motion would be a result of the Romano-Ward syndrome and not damage from ischaemic heart disease. The only patient with exertional chest pain was found to have normal coronary arteries at cardiac catheterisation.

Only members of family 2 had detectable left ventricular defects. These patients also had reduced ejection fractions, more pronounced in the two with symptoms.

The differences between the two families in the electrocardiographic morphology, and in the finding of left ventricular abnormalities in family 2 , but not in family 1 , suggest that Romano-Ward syndrome is a heterogeneous 
disorder. Indeed, within family 2 , it may be necessary to have a specific degree of abnormality of the left ventricular muscle wall before the manifestation of symptoms. It will be interesting to see if subject V.2 develops symptoms in the future, and if so, if there has been any deterioration in left ventricular function.

The subtle abnormalities of right ventricular phase are interesting but not uniform either within or between families. The presence of an abnormal right ventricular phase did not relate to the presence or frequency of symptoms.

Perhaps our understanding of this interesting syndrome is limited because it is a collection of syndromes within a syndrome, all with slightly different pathology but similar clinical presentations.

1 Ward OC. A new familial cardiac syndrome in children Fournal of the Irish Medical Association 1964;54:103-6.

2 Romano C. Congenital cardiac arrhythmia. Lancet 1965;i:658-9.

3 Mathews EC, Blount AW, Townsend JI. QT prolongation and ventricular arrhythmias, with and without deafness, in the same family. Am $₹$ Cardiol 1972;29:702-11.

4 Schwartz PJ, Periti M, Malliani A. The long QT syndrome. Am Heart f 1975;89:378-90.

5 Bharati S, Dreifus L, Bucheleres G, et al. The conduction system in patients with a prolonged QT interval. $\mathcal{F} A m$ Coll Cardiol 1985;6:1110-9.

6 James T, Froggatt P, Atkinson WJ, et al. Observations on the pathophysiology of the long QT syndromes with special reference to the neuropathology of the heart. Circulation 1978;57:1221-31.

7 Bazett HC. An analysis of the time-relations of electrocardiograms. Heart 1920;7:353-70.

8 Lepeschkin $\mathrm{E}$. The measurement of the QT interval of the electrocardiogram. Circulation 1952;6:378-88.

9 Evans NTS, Keyes WJ, Smith D, et al. The Aberdeen Mark II single-photon-emission tomographic scanner: specification and some clinical specifications. Phys Med Biol 1986;31:65-78.

10 Norton MY, Walton S, Evans NTS. Gated cardiac tomography. Eur 7 Nucl Med 1988;14:472-6.

11 Cross SJ, Lee HS, Norton MY, Walton S. Three-dimensional tomographic imaging of the right ventricle sional tomographic imaging of the righ

12 Cross SJ, Lee HS, Norton MY, et al. Three dimensional imaging of the right ventricle using blood pool tomography. European Radiology 1992;2:95-8.

$13 \mathrm{Kahn}$ NK, Sisson J, Vinik A. QT interval prolongation and sudden cardiac death in diabetic autonomic neuropathy. $\mathcal{F}$ Clin Endocrinol Metab 1987;64:751-4.

14 Yanowitz F, Preston J, Abildskov JA. Functional distribution of right and left stellate innervation to the ventricles. Circ Res 1966;18:416-28.

15 Schwartz PJ, Locati E. The idiopathic long QT syndrome: pathogenetic mechanisms and therapy. Eur Heart $\mathcal{f}$ 1985;6(suppl D): 103-14.

16 Atwell D, Lee JA. A cellular basis for the primary long QT syndromes. Lancet 1988;i:1136-9.

17 Moothart RW, Pryor R, Hawley RL, Clifford NJ, Blount SG. The heritable syndrome of prolonged QT interval, syncope, and sudden death. Electron microscopic syncope, and sudden death. E
observations. Chest 1976;70:263-6.

18 Bexton RS, Vallin HO, Camm AJ. Diurnal variation of the QT interval-influence of the autonomic nervous system. Br Heart $\mathcal{F}$ 1986;55:253-8.

19 Stern S, Eisenberg S. The effect of propranolol (Inderal) on the electrocardiogram of normal subjects. Am Heart f 1969;77:192-5.

20 Milne JR, Ward DE, Spurrel RAJ, Camm AJ. The long QT syndrome: effect of drugs and left stellate ganglion block. Am Heart f 1982;104:194-8.

21 Yanagida $\mathrm{H}$, Kemi C, Suwa $\mathrm{K}$. The effects of stellate ganglion block on idiopathic prolongation of the QT interval with cardiac arrhythmia (the Romano-Ward vyndrome). Anesth Analg 1976;55:782-7.

22 Wilmer CI, Stein B Morris DC Atrioventricular pacemaker placement in Romano-Ward syndrome and maker placement in Romano-Ward syndrome and recurrent torsad

23 Jackman WM, Friday KJ, Anderson JL, Aliot EM, Clark $M$, Lazzara $R$. The long QT syndromes: a critical review, new clinical observations and a unifying hypothesis. Prog Cardiovasc Dis 1988;31:115-72.

24 Metcalfe MJ, Norton MY, Walton S. Improved sensitivity of tomography in comparison to planar radionuclide ventriculography in myocardial infarction [abstract]. Nucl Med Commun 1989;10:206-7.

25 Metcalfe MJ, Norton MY, Jennings $K$, Walton S. An improved method of detecting inferior wall motion abnormalities by the use of tomographic scintigraphy. Eur Heart f 1989;10(suppl):322. 